\title{
White bear everywhere: Exploring the boundaries of the attentional white bear phenomenon
}

\author{
Aya Lahav • Tal Makovski $\cdot$ Yehoshua Tsal
}

Published online: 10 February 2012

(C) Psychonomic Society, Inc. 2012

\begin{abstract}
Some failures of selective attention may be explained by the attentional white bear (AWB) phenomenon Tsal \& Makovski (Journal of Experimental Psychology: Human Perception and Performance 32:351-363, 2006), which indicates that prior knowledge of a distractor location causes attention to be actively allocated to it. The AWB effect is demonstrated in a task that includes infrequent trials that involve two simultaneous dots embedded among flanker trials. The dot positioned at an expected distractor location is perceived as appearing before the dot at an expected empty location, indicating that attentional resources have initially been allocated to the expected distractor locations. The main goal of this study was to explore the boundaries of the AWB phenomenon by imposing perceptual, memory, and sensory constraints on the flanker task. The results showed that the AWB effect was obtained even when additional constraints severely taxed the information-processing system. We propose that a mandatory mechanism guides a fixed minimal amount of attention to expected distractor locations in a topdown manner.
\end{abstract}

Keywords Selective attention - Attentional white bear effect - Visual attention - Distractor processing - Distractor inhibition · Flanker task $\cdot$ Temporal order judgment

\footnotetext{
A. Lahav $\cdot$ Y. Tsal $(\bowtie)$

Department of Psychology, Tel Aviv University,

Ramat Aviv,

Tel Aviv 69978, Israel

e-mail: jehoshua@freud.tau.ac.il

A. Lahav

e-mail: aya.lahav@gmail.com

T. Makovski

University of Minnesota,

Minneapolis, MN, USA
}

The world we view is composed of multiple stimuli, but only a portion of these stimuli can be selected for further processing. Many theories of attention (e.g., Cave \& Wolfe, 1990; Treisman \& Gelade, 1980; Wolfe, 1994) stipulate that attentional selection is accomplished through the selection of a region in space and the processing of stimuli within that region. However, allocating attention to a clearly distinguishable region is far from perfect, and irrelevant stimuli from outside the selected region are often processed as well. Such a failure of selective attention has most commonly been demonstrated in the flanker task (e.g., Eriksen \& Eriksen, 1974). In this task, participants are asked to attend to a central target letter and ignore two flanking distractor letters. The flanking letters could be mapped onto a response that is either congruent or incongruent with the target's response. Typically, a response compatibility effect with the identity of the distractors is found, showing longer reaction times (RTs) when the target's and the distractor's responses are incongruent than when they are congruent, indicating that the distractors have been processed. Because the central target has a processing advantage over the peripheral distractors, and because the target and distractor positions are clearly distinguished and known in advance, such distractor interference is surprising, as one might expect attention to remain consistently, and continuously, fully allocated to the target throughout the task.

Tsal and Makovski (2006) pointed out an inherent discrepancy in the flanker task, as well as in other basic tasks of selective attention. Such tasks call for participants to attend to the target and ignore the distractors. These instructions require the participants to discern the target and distractor locations and differentiate them from other, unspecified, empty locations. It is possible that the very specification of these stimulus locations initiates activation that begins with attentional allocation to these spatial regions. This activation 
is in line with the instructions to attend to the target, but it is incompatible with the request to ignore the distractors. Thus, the instructions given in the flanker task may initiate an active allocation of attention toward both the target and distractor locations, which enhances the processing of the target but hampers the ability to ignore the distractors.

In order to examine the possibility that distractor locations become attended due to their expectancy, Tsal and Makovski (2006) used a flanker task in which the distractors were placed diagonally at either the top left and bottom right or the top right and bottom left positions from the central target. The locations of the distractors remained fixed throughout the block, and could therefore be anticipated. On a minority of unexpected trials, instead of a flanker display, two dots appeared simultaneously. One of the dots occupied the location in which a distractor always appeared in the flanker trials, and the other dot occupied a location that was always empty during the flanker trials. Since the flanker trials were much more frequent than the two-dot trials, and since the two trial types were randomly intermixed, participants strategically should have always expected and prepared themselves for a flanker trial. Therefore, it could be maintained that when the two-dot trials were presented, one dot was positioned at an expected distractor's location, and the other dot appeared at an expected empty location. Participants were asked to determine which of the two dots had appeared first. Stelmach and Herdman (1991) showed that the perceived temporal order of two simultaneous stimuli is affected by attention; the attended one is consistently perceived as appearing before the unattended one. The shooting-line illusion (Hikosaka, Miyauchi, \& Shimojo, 1993) also shows a similar effect: when one end of the line is attended, the line is perceived as extending from the attended end toward the unattended one. Tsal and Makovski's results showed that the dot at the expected distractor location was perceived as appearing before the dot at the expected empty location, indicating that attention was allocated to the expected distractor location. Similarly, when the authors presented a horizontal line instead of the two dots, the line appeared to extend from the expected distractor location toward the expected empty location. The fact that these attentional biases were found while participants prepared for a flanker display indicates that attention was allocated toward the distractor location prior to stimulus onset on the basis of topdown expectations. This effect was named the attentional white bear (AWB) phenomenon.

In order to eliminate the alternative possibility that attention was drawn to the distractor location due to priming of that location by the stimulus in the previous trial, the same experiment was also conducted with blocks in which the distractor position varied randomly. The results showed that the distractor location in the preceding trial did not bias attention toward that location when the distractor location could not be anticipated in the upcoming trial, thereby supporting the top-down interpretation of the AWB effect. Tsal and Makovski (2006) also ruled out the possibility that the results were due to a response bias inherent in the forced choice design, causing participants to choose from two simultaneously perceived dots the one that appeared in the location of the expected distractor location. They adopted a manipulation proposed by Shore, Spence, and Klein (2001) to eliminate such biases in the temporal judgment taskpresenting one white and one yellow dot (randomly positioned in the left and right locations) and asking their participants to indicate the color of the dot that appeared second. This manipulation dissociated the response and the expected distractor location. The results showed that the dot appearing at the location of the expected distractor was perceived as appearing before the dot at the expected empty location, thus discouraging the notion that the AWB effect is due to response biases rather than reflecting a genuine perceptual difference.

Another possibility ruled out by Tsal and Makovski (2006) was that the top distractor was not fully unattended because it served as a cue to the temporal judgment task. Participants could have allocated attention to the top two diagonal positions in anticipation of the dot or line displays, in spite of their infrequency and in spite of the potential interference of the frequently appearing distractor. One should note that this possibility could not fully account for the results, because the dot in the expected distractor location was perceived as occurring before the dot in the expected empty location, although both positions were equally relevant to these tasks. To examine the above alternative possibility, Tsal and Makovski placed a congruent distractor in one position and an incongruent distractor at the other. If participants attended to the top distractor more than the bottom one, greater interference in the flanker task should be obtained when the top distractor was incongruent (and the bottom one congruent) than when the top distractor was congruent (and the bottom one incongruent). The results showed similar responses to both types of flanker displays, thus refuting the possibility that the AWB effect was obtained because participants deliberately paid more attention to the location of the distractor coinciding with one of the dots in the temporal judgment task.

In order to further validate the notion that the temporal judgment task is a reliable measure of attentional allocation, Tsal and Makovski (2006) showed that locations that are expected to be highly attended generate temporal judgment effects in their favor. Participants matched two targets in either the absence or presence of distractors. On unpredictable trials, two dots appeared, one in the expected target location and one in either the expected distractor location or an expected empty 
location. Results showed a temporal order preference for the target over the distractor location, as well as for the target over the empty location. Since the target is assumed to be highly attended, these results further substantiated the use of the temporal order judgment for assessing the differential allocation of attention over the visual field.

Tsal and Makovski's (2006) findings suggest that the AWB effect is based on a mandatory "process-all" mechanism, because it occurs in spite of task goals to ignore distracting locations. However, this effect was demonstrated while using a flanker task, which is a relatively easy task with low demands. It is possible that the AWB effect is not general, but rather is restricted to tasks of low difficulty. The purpose of the present study was to investigate whether the AWB effect is specifically related to low task demands, which leave spare attentional resources to be allocated to all display stimuli, or whether it is more obligatory in nature, thus persisting even when attentional resources are severely constrained. If the AWB effect is mediated by a flexible mechanism that takes the expected difficulty of the upcoming task into account and allocates resources to the distractors accordingly, then the effect should be reduced or eliminated as task difficulty increases. On the other hand, if the effect is obligatory, then a fixed amount of attention is always allocated toward the expected distractor locations, regardless of the expected task demands. In order to examine these questions, we applied a comprehensive inspection of the possible boundaries of the AWB effect by introducing additional perceptual, memory, and sensory constraints, each burdening a different part of the process. Sensory constraints disrupt the early stages of processing by degrading the amount of available input. This reduction in available data may require that more resources be allocated to each degraded stimulus in order to improve its processing. Perceptual constraints increase task difficulty at the stage of perceptual identification, limiting the ability to segregate and identify the stimulus. Such constraints may also require that additional attentional resources be allocated to the processing of the various stimuli, possibly affecting resource distribution. Finally, the memory constraints increase the cognitive demands, making it more difficult to keep task priorities and to suppress interference from irrelevant stimuli. Introducing additional constraints will cause a reduction in attentional resources, which may lead to more effective prioritization of display stimuli, with a higher preference for the target over the distractor. This could reduce the AWB effect, because processing of the target will take precedence, causing all spare attention to be allocated to the target location. The opposite outcome is also possible, as the increase in task demands may further motivate participants to ignore the anticipated distractor, which would increase the initial allocation of attention toward the expected distractor location. A third possibility is that the magnitude of the AWB effect will not be affected by increased task demands, because the effect is a process-all mechanism. According to this view, attention is guided to all expected stimulus locations, regardless of task relevance, difficulties, or participants' intentions, and a fixed amount of resources are always allocated to the distractor location.

\section{Experiment 1}

The most commonly used manipulation of task difficulty has been the manipulation of perceptual load. Perceptual load is defined as an increase in the demands of perceptual identification and has typically been manipulated by increasing the number of neutral items surrounding the target. This manipulation is assumed to require that maximal attentional resources be applied in order to segregate and identify the target, so that no resources are left over to be captured by the distractors (see, e.g., Lavie, 1995).

In the present experiment, we used a classic manipulation of perceptual load in which participants responded to a central target while trying to ignore a peripheral distractor. In the low-load condition the target appeared alone, and in the high-load condition it was surrounded by three neutral letters. The additional neutral letters in the high-load condition are thought to produce perceptual constraints on target processing. Tsal and Benoni (2010) argued that it is impossible to completely dissociate perceptual resource limitations from sensory or memory constraints. Hence, the manipulation in the present experiment might place an additional burden on the sensory and memory demands. The extra neutral letters around the target might degrade the sensory processing of the target letter because of possible masking by the adjoining stimuli. This manipulation might also increase memory demands by requiring that each additional central stimulus be compared to the internal representation of the target before it is rejected. However, the purpose here was not to completely dissociate the various types of burdens, but rather to attempt a variety of manipulations in order to assess the boundary conditions of the AWB effect.

\section{Method}

Participants The participants in all experiments were undergraduates from Tel Aviv University who participated in the experiments as part of a course requirement. All participants had normal or corrected-to-normal vision.

A group of 26 participants ( 22 female, 4 male), ranging in age from 19 to 33 years, with a mean age of 22 , took part in Experiment 1. 
Apparatus All experiments were conducted in a dimly lit room. Stimulus presentation and data collection were controlled by a Pentium 1.8-GHz processor, and the stimuli were presented on a Sony CPD 200SX monitor (17-in.) using a screen resolution of $600 \times 800$ pixels. Stimuli were presented via the Authorware software (Adobe Systems Inc.). A chinrest was used to stabilize viewing distance at $40 \mathrm{~cm}$, so that $1 \mathrm{~cm}$ on the display would represent $1.43 \mathrm{deg}$ of visual angle.

Stimuli and procedure Participants were presented with two conditions. In the low-load condition, the stimulus display contained a central target and two diagonal distractors. In the high-load condition, three central neutral letters were added to the display. Participants were instructed to respond as quickly and as accurately as possible to the central target while ignoring the distractors. They pressed the "L" key with their right index finger when the target was the letter $\mathrm{S}$, and the "A" key with their left index finger when the target was the letter $\mathrm{K}$. The target letter was diagonally flanked by two distractors that could either be identical to (i.e., congruent trials) or different from (i.e., incongruent trials) the target. The two possible target letters were equally frequent and were randomly intermixed. For each target letter, the two distractors were randomly intermixed. Each of the letters (target, distractor, and neutral) was white and subtended $0.57 \mathrm{deg}$ in height and $0.43 \mathrm{deg}$ in width. The distractors were centered $2.3 \mathrm{deg}$ from the center of the display. The flanker displays in the high-load condition consisted of four central stimuli- one target letter and three neutral letters - centered $0.85 \mathrm{deg}$ from the center and positioned in the shape of a diamond. Each neutral central letter had an equal chance of being an $\mathrm{N}$ or an $\mathrm{O}$. The target letter had an equal chance of appearing in each of the four central locations. In the low-load condition, the three central nontargets were replaced by three white horizontal lines that subtended $0.43 \mathrm{deg}$ in height and $0.14 \mathrm{deg}$ in width.

Each condition comprised two fixed blocks in which the distractors occupied the same diagonal positions. In one block, they appeared at the top left and bottom right positions, and in the other, they appeared at the top right and bottom left positions. The participants were notified about the locations of the distractors at the start of each block. The order of the two blocks was randomized within each condition, and the order of the two conditions was randomized across participants. Each block comprised 112 diagonal flanker displays and 38 two-dot displays. The two display types were randomly intermixed, with the constraint that the two-dot displays were never presented on two consecutive trials. The two-dot displays consisted of two dots presented simultaneously, one in the location of the center of the top left distractor, and the other in the location of the center of the top right distractor. Each dot subtended $0.064 \mathrm{deg}$ in diameter. The distance between the dots in the two-dot display was 3.15 deg of visual angle. Participants were required to indicate which of the dots appeared first by pressing the " $\mathrm{K}$ " key with their right index finger, for the right dot, and the "S" key with their left index finger, for the left dot. Only accuracy was emphasized in this task.

Each trial began with a 500 -ms fixation cross $(0.28 \times$ $0.28 \mathrm{deg}$ ), followed by a 500 -ms blank interval, followed by the stimulus display, which was presented until response (see Fig. 1). Trials were separated by another $500-\mathrm{ms}$ interval. Each condition began with 25 practice trials, which had a load similar to that of the experimental trials in that condition. The distractors' location in the practice trials varied randomly. Auditory feedback was given on accuracy in the flanker task during the practice trials. Written instructions regarding the upcoming distractors' locations were given at the start of each block within a condition.

Results and discussion

Flanker task A Perceptual Load (high vs. low) $\times$ Congruency (congruent vs. incongruent) repeated measures ANOVA was carried out on the accuracy data. The results did not reveal any significant effects $[F(1,25)=1.05, p=$ .315 , for load; $F(1,25)=1.65, p=.21$, for congruency; and $F(1,25)=0.18, p=.67$, for the interaction].

Incorrect responses and responses deviating by more than two standard deviations from the mean were removed from the RT analyses. A similar ANOVA performed on mean RTs showed significant effects for both load and congruency. The responses in the high-load condition were slower than those in the low-load condition $[F(1,25)=20.13, p<.001]$, and congruent displays were responded to faster than incongruent ones $[F(1,25)=7, p=.014]$. The interaction between load and congruency was not significant $[F(1,25)=0.18, p=.68]$. Table 1 shows the RTs and accuracy rates in all experiments.

The flanker task results showed a congruency effect in the expected direction, indicating that the distractor stimuli were attended and processed. The results also showed that high perceptual load indeed did make the task more difficult, as was evident from the significantly longer RTs in the high-load condition. The lack of a Load $\times$ Congruency interaction, however, is inconsistent with perceptual-load theory, which predicts greater distractor interference under low-load than under high-load conditions (Lavie, 1995). This is not the first reported difficulty in replicating the expected perceptual-load results (see, e.g., Eltiti, Wallace, \& Fox, 2005; Miller, 1991; Tsal \& Benoni, 2010; Wilson, Muroi, \& MacLeod, 2011). Most important for the present study is that the load manipulation produced a substantial increase in overall RTs, indicating that task difficulty was indeed increased in the high-load condition. 
Fig. 1 Example of an incongruent flanker trial followed by a two-dot trial in the high- and low-load conditions in Experiment 1

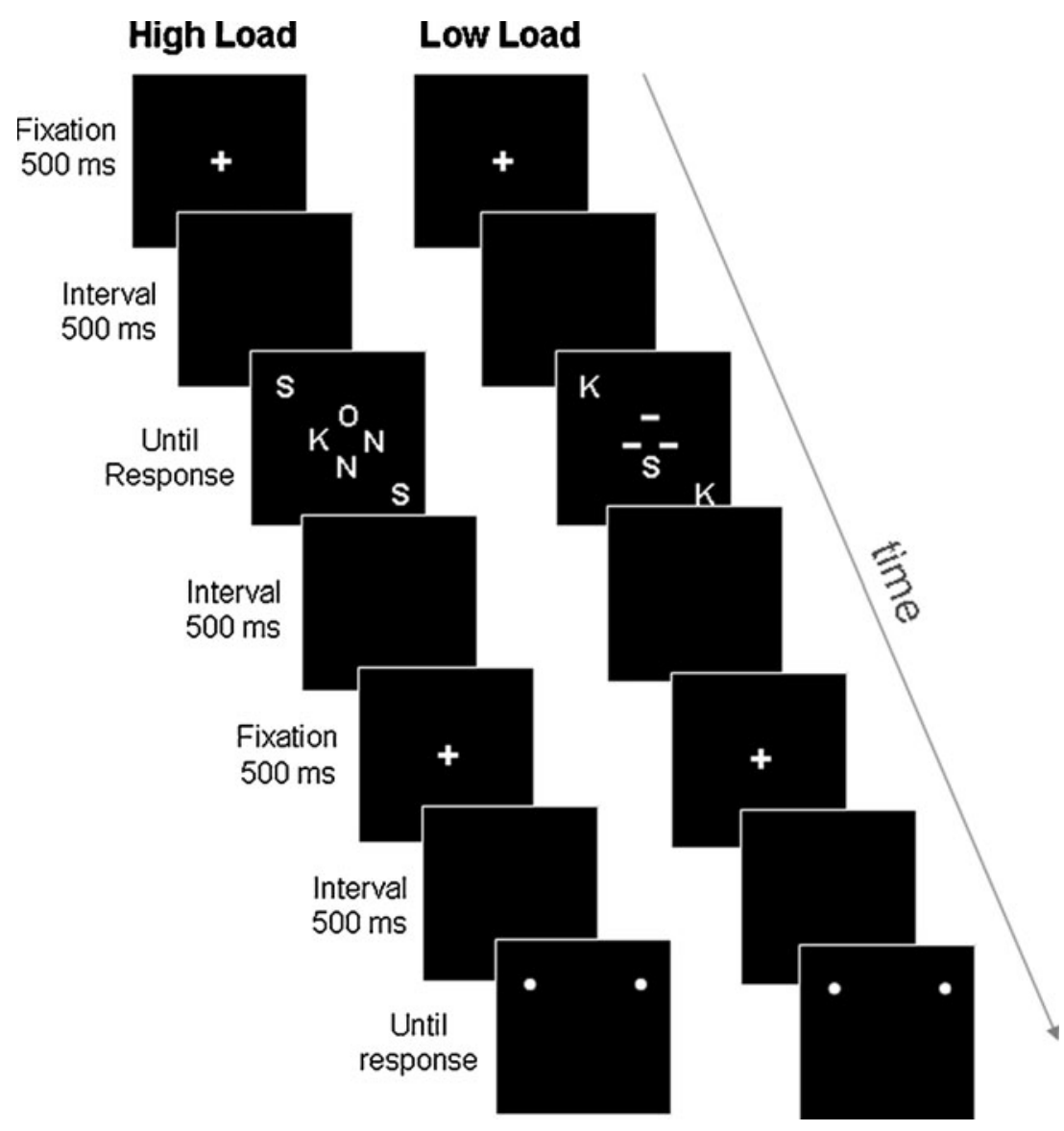

$[F(1,25)=7.97, p=.009]$ : Participants produced more "left" responses when anticipating a distractor in the top left position rather than in the top right position. This result replicates the basic AWB effect reported by Tsal and Makovski (2006) and shows that expecting a distractor to appear at a certain location results in perceiving a dot at that location as appearing before a dot that appears at an unexpected location prior to stimulus onset.

There was no difference between the proportions of "left" responses in the high- and low-load conditions $[F(1,25)=$ $0.1, p=.76]$. The interaction between these two factors was also not significant $[F(1,25)=0.004, p=.95]$, indicating that the magnitude of the effect was the same under both load conditions. Hence, increasing the difficulty of the relevant

Table 1 Means reaction times (RTs; with standard deviations), in milliseconds, and mean accuracy rates for the congruent and incongruent flanker displays of Experiments 1-3

\begin{tabular}{|c|c|c|c|c|c|c|c|}
\hline & & \multicolumn{2}{|c|}{ Perceptual (Exp. 1) } & \multicolumn{2}{|c|}{ Memory (Exp. 2) } & \multicolumn{2}{|c|}{ Sensory + Memory (Exp. 3) } \\
\hline & & High Load & Low Load & High Load & Low Load & High Load & Low Load \\
\hline \multirow[t]{2}{*}{ Congruent } & RTs $(S D)$ & 772 (187) & $661(145)$ & $758(128)$ & $643(129)$ & $865(205)$ & $771(237)$ \\
\hline & Accuracy rates $(\%)$ & 97 & 97 & 97 & 97 & 95 & 96 \\
\hline \multirow[t]{2}{*}{ Incongruent } & RTs $(S D)$ & $785(189)$ & $679(137)$ & $839(165)$ & $670(132)$ & 899 (236) & $791(244)$ \\
\hline & Accuracy rates $(\%)$ & 97 & 96 & 93 & 95 & 92 & 96 \\
\hline
\end{tabular}




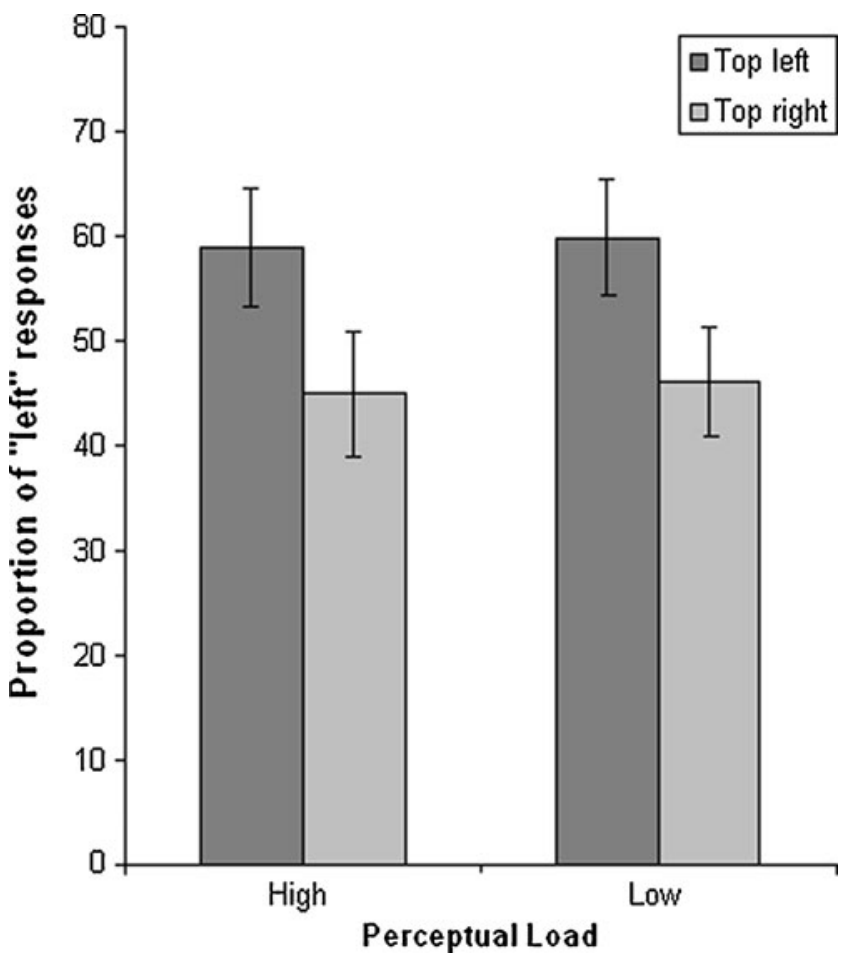

Fig. 2 Mean proportions of "left" responses (perceiving the left dot before the right dot) in the high- and low-load conditions in Experiment 1

task did not diminish the allocation of attention to irrelevant, interfering distractors.

One possible criticism of the present results is that, since the locations of the distractors were kept fixed throughout the block, the distractor location effect might not represent top-down anticipation, but might actually derive from priming of the distractor location by the previous trial. Tsal and Makovski (2006) ruled out this possibility by showing in several experiments that attention was not allocated to a location primed by the previous trial when the upcoming distractor location was not known. In order to ascertain that top-down expectations were the only contributors in the present study, we conducted the same experiment again, this time with the locations of the distractor diagonals switching randomly throughout the block. We compared the temporal judgment biases between trials preceded by a distractor in the left location and those preceded by distractor in the opposite location. If the temporal judgment effect is top-down driven, the AWB effect would be expected to be eliminated, since participants could not anticipate the distractor location on the next trial. Indeed, when 11 new participants were run on the perceptual-load experiment with randomly determined distractor locations, the distractor location effect was completely abolished $[F(1,10)=0.06, p=.8]$, indicating that the AWB effect disappears when participants cannot anticipate the location of the upcoming distractor and allocate attention accordingly.

\section{Experiment 2}

Experiment 1 showed that adding perceptual constraints to the flanker task did not affect the magnitude of the AWB effect. In the present experiment, we attempted to explore the possible influence of memory constraints on this effect. Working memory is a higher-order function that maintains the mental representation of a stimulus even when the stimulus is not currently being presented (Baddeley, 1986). The interaction between working memory and visual attention is well documented (Downing, 2000; Makovski, Swallow, \& Jiang, 2011; Olivers, 2009; Soto, Heinke, Humphreys, \& Blanco, 2005). For instance, it has been claimed that maintaining a template of the target item elicits the selection of that item among various irrelevant stimuli (e.g., Desimone \& Duncan, 1995). Therefore, increasing working memory demands might interfere with selection because of the difficulty of maintaining processing priorities.

Initial pilot results indicated difficulties in performing the temporal judgment task in conjunction with the flanker task. We therefore introduced an additional short practice block that solely included the temporal judgment task. In the short dotpractice block, the dots actually appeared separated in time. On the first eight trials, the separation was relatively large $(50 \mathrm{~ms})$, and on the following eight trials, it was shorter but still noticeable $(30 \mathrm{~ms})$. Auditory feedback indicated erroneous answers to the participants. The purpose of this practice block was to sharpen participants' perception of temporal order.

\section{Method}

Participants A group of 11 participants (9 female, 2 male), ranging in age from 21 to 26 years, with a mean age of 23 , took part in Experiment 2.

Apparatus The apparatus was the same as in the first experiment, except that the chinrest was placed at a distance of $60 \mathrm{~cm}$ from the display, so that $1 \mathrm{~cm}$ on the display represented 0.95 deg of visual angle.

Stimuli and procedure The method was similar to that of Experiment 1, except for the following changes. Participants were presented with two conditions of memory load, high and low. Each condition comprised two 200-trial blocks. Each of these blocks consisted of 160 diagonal flanker and 40 two-dot displays, which were randomly intermixed. Each trial began with a memory display, followed by a flanker or dot display. The high-load condition consisted of three letters in the memory display, whereas the memory display in the low-load condition consisted of one letter and two hash signs ("\#”). The memory display letters were randomly chosen from 24 possible letters (excluding the letters $\mathrm{O}$ and I). No letter was repeated within the same (high-load) 
memory display. All of the stimuli (primes, target, and distractors) were white and subtended $0.57 \mathrm{deg}$ in height and $0.38 \mathrm{deg}$ in width. The three items in the memory display were presented at the center, with a center-tocenter distance between adjoining items of $0.57 \mathrm{deg}$. The single letter in the low-load condition randomly appeared in each of the three possible locations. The flanker displays consisted of a central target and two distractors placed at the diagonal positions. The center-to-center distance between the target and each distractor was $1.15 \mathrm{deg}$ of visual angle. Participants were instructed to indicate whether the target in the flanker display was one of the letters from the memory display. They pressed the "A" key with their left index finger when the target was a letter from the memory display and the "L" key with their right index finger when the target was not. Half of the distractors were congruent with the target - that is, belonged to the same response category (e.g., both the target and distractor letters appeared in the memory display) - and half were incongruent - that is, belonged to the opposite response category (e.g., the target did not appear in the memory display, but the distractor letters did). The two possible trial types (congruent and incongruent) were equally probable. In order to equate between conditions, when the target was a letter from the memory display in a congruent trial, the distractors were the same letter as the target in both the high- and low-load conditions. Each trial began with a memory display presented for $500 \mathrm{~ms}$, followed by a $500-\mathrm{ms}$ blank interval, a 500-ms fixation cross, another blank interval of $500 \mathrm{~ms}$, and finally the stimulus display. The stimulus display remained on screen until a response. A 500-ms blank interval separated trials (see Fig. 3).

Each session began with 16 practice temporal judgment trials, in which one dot appeared before the other. For the first 8 trials, the lag between the two dots was $50 \mathrm{~ms}$, and in the following 8 trials, the lag was reduced to $30 \mathrm{~ms}$. During this part of practice, auditory feedback was given on accuracy. At the end of these trials, the participants were informed that from this stage on, the temporal judgment trials would appear randomly intermixed within the flanker trials. The initial practice then continued with 12 introductory practice trials with random distractor positions and the same load as the upcoming experimental condition. An additional 12-trial practice with random distractor positions was given before the start of the second condition, with load displays taken randomly from within the upcoming condition. Auditory feedback was given on accuracy for the flanker task during the practice trials. Written instructions regarding the upcoming distractors' locations were given at the start of each block within the condition.

\section{Results and discussion}

Flanker task A Memory Load (high vs. low) $\times$ Congruency (congruent vs. incongruent) repeated measures ANOVA
Fig. 3 Example of a flanker trial in the high-load (and here, incongruent) and low-load (here, congruent) conditions of Experiment 2

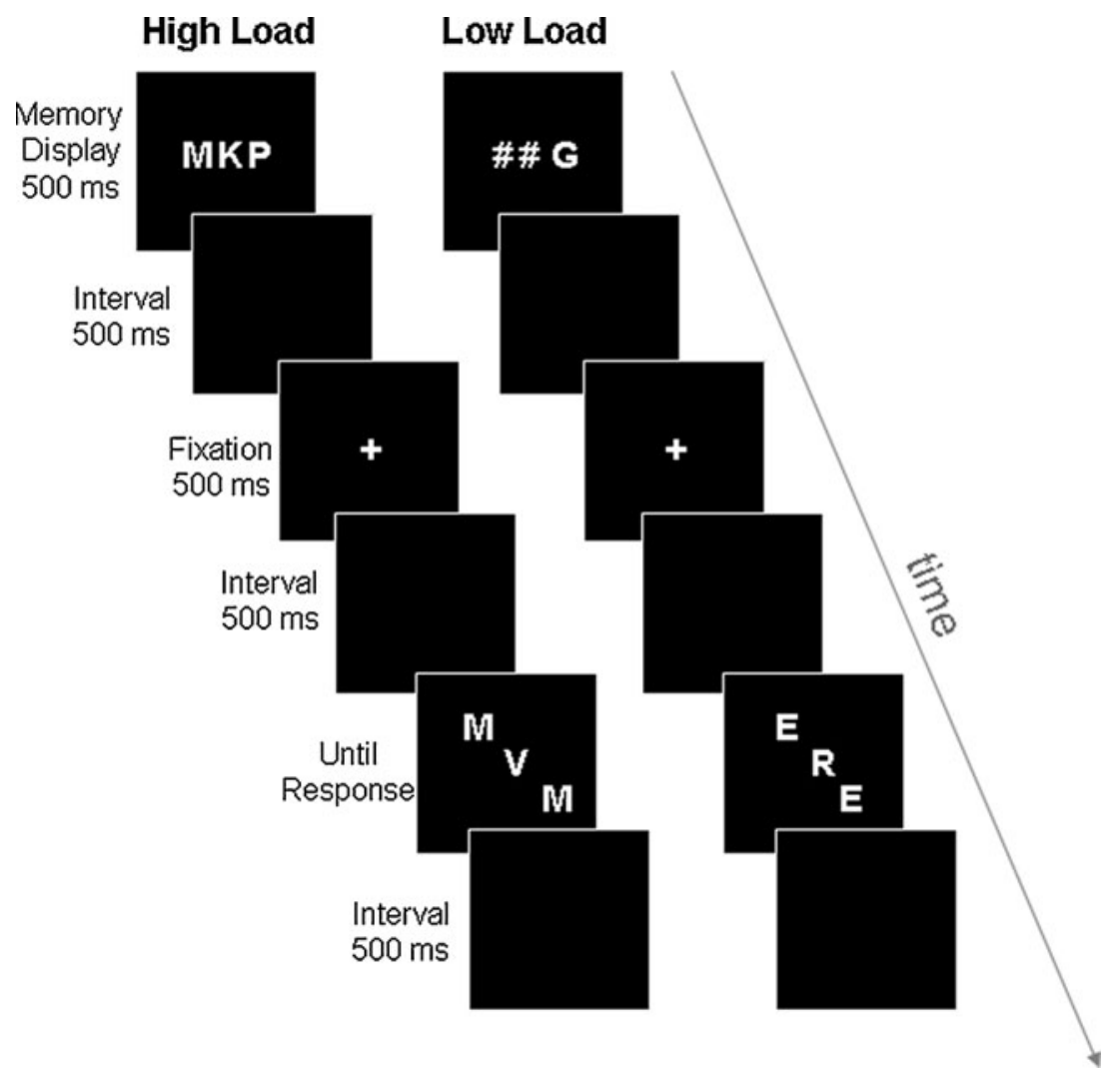


carried out on the accuracy data revealed a borderline congruency effect $[F(1,10)=4.57, p=.058]$. Neither the effect of load $[F(1,10)=1.41, p=.26]$ nor the Load $\times$ Congruency interaction $[F(1,10)=0.2, p=.66]$ reached significance.

Incorrect responses and responses deviating by more than two standard deviations from the mean were removed from the RT analyses. A similar Memory Load (high vs. low $) \times$ Congruency (congruent vs. incongruent) ANOVA was performed on the mean RTs. The results revealed that all effects were significant: Responses in the low-load condition were faster than those in the highload condition $[F(1,10)=14.75, p=.003]$. Responses for congruent trials were faster than those for incongruent trials $[F(1,10)=33.08, p<.001]$. Also, the congruency effect was significantly larger in the high-load condition than in the low-load condition $[F(1,10)=5.38, p=.043]$.

The main condition effect served as a manipulation check and confirmed that increasing the number of letters to memorize indeed did lead to longer response latencies. The results also showed an interaction, which is in line with findings from previous studies that have shown that congruency effects increase when memory constraints are added (Lavie \& de Fockert, 2005; Lavie, Hirst, de Fockert, \& Viding, 2004).

The design of the present experiment necessitated the introduction of a potential response bias in the flanker task. Since the low-load condition had a single letter in the memory display, all congruent trials in which the target letter matched the memory display would have to be composed of the same letters in the diagonal. This made up half of the congruent trials in this condition. The same-letter diagonal could possibly serve as a cue, because participants would not need to process the identity of the letters in order to reach a correct response. Because of this possible bias, we made sure that the same type of congruent trials in the highload conditions were also composed of same-letter diagonals. Thus, if such a bias indeed took place in the flanker task, it was equally present in both the high- and lowmemory-load conditions.

Temporal judgment task A Memory Load (high vs. low) $\times$ Distractor Location (top left vs. top right) repeated measures ANOVA performed on the proportions of "left" responses (see Fig. 4) showed only a significant effect of distractor location $[F(1,10)=6.2, p=.032]$ : Participants produced more "left" responses when anticipating a distractor in the top left position than in the top right position. There was no difference between the proportions of "left" responses in the high- and low-load conditions $[F(1,10)=0.1, p=.76]$, nor was there a significant Load $\times$ Distractor Location interaction $[F(1,10)=2.01, p=.19]$.

The results of the temporal judgment task replicated those of the previous experiment: Attention was allocated to the expected distractor locations, producing an AWB

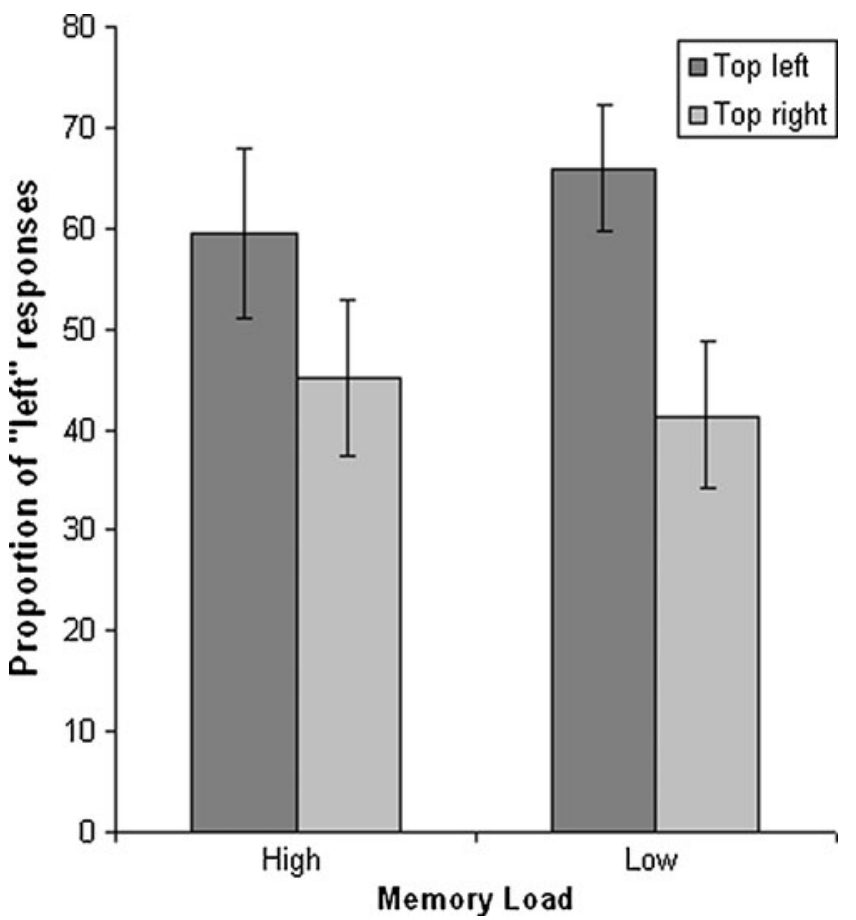

Fig. 4 Mean proportions of "left" responses (perceiving the left dot before the right dot) in the high- and low-load conditions of Experiment 2

effect. The magnitude of this effect did not change, even with a substantial increase in memory load.

\section{Experiment 3}

The results of the preceding experiments showed that increasing task difficulty by either perceptual or cognitive means failed to influence the AWB effect, as the temporal judgment bias preserved its magnitude under all of the above manipulations. These results suggest that the AWB effect is highly rigid and mandatory, so that the allocation of attention to interfering distractors is not diminished even when substantial attentional resources are required for target processing under highly constrained conditions.

However, it is also possible that the additional constraints in our previous tasks were simply not severe enough to force participants to focus all attentional resources on the target, thereby diminishing the AWB effect. Therefore, in our final experiment we attempted to create even more extreme circumstances for the flanker task, by combining sensory and memory constraints. Sensory limitations, degrading the quality of the target, were created by reducing its brightness and size and presenting a subsequent mask. This manipulation interferes with the early processing of stimuli by constraining the available data or the time to process that data and/or consolidate them into memory (Norman \& Bobrow, 1975). Consequently, each limitation might impair 
target identification (because information is degraded) as well as target selection (because the ability to differentiate between the stimulus items is reduced). A combination of both sensory and memory constraints would simultaneously burden both the visual and cognitive systems, requiring vast amounts of processing resources. We predicted that if the AWB effect is somewhat penetrable to the attentional demands of the task, it would be evident in a diminished AWB effect under these extreme conditions.

\section{Method}

Participants A group of 16 participants (14 female, 2 male), ranging in age from 18 to 26 years, with a mean age of 22 , completed the experiment. Six of these participants were excluded due to less than $80 \%$ correct responses.

Stimuli and procedure The method was similar to that of Experiment 2, except for the following changes. Participants were presented with two conditions: difficult and easy. The target letter in the easy condition was white and subtended $0.57 \mathrm{deg}$ in height and $0.38 \mathrm{deg}$ in width. The target letter in the difficult condition was dark gray (color $16 \times 12$ in the palette) and subtended 0.29 deg in height and 0.19 deg in width. The target letter in the difficult condition was followed by a hash sign (\#) mask with a similar color and dimensions. The target and distractors appeared simultaneously in the display, and the distractors remained on screen for $200 \mathrm{~ms}$. In the easy condition, the target remained on screen for $100 \mathrm{~ms}$, while in the difficult condition, the target remained for $100 \mathrm{~ms}$, followed by a 100-ms presentation of the mask. The screen remained blank after the stimuli disappeared until the participant made a response. A 500-ms interval separated trials (see Fig. 5).

Each session began with 16 practice temporal judgment trials in which one dot appeared before the other, followed by 15 practice trials from the easy condition, and then by 15 trials from the difficult condition.

Results and discussion

Flanker task An overall repeated measures Condition (difficult vs. easy) $\times$ Congruency (congruent vs. incongruent) ANOVA was carried out on the accuracy data. The analysis revealed a borderline effect for condition $[F(1,9)=4.4, p=$ .065], showing more erroneous responses in the difficult
Fig. 5 Example of a flanker trial in the difficult (here, congruent) and easy (here, incongruent) conditions of Experiment 3

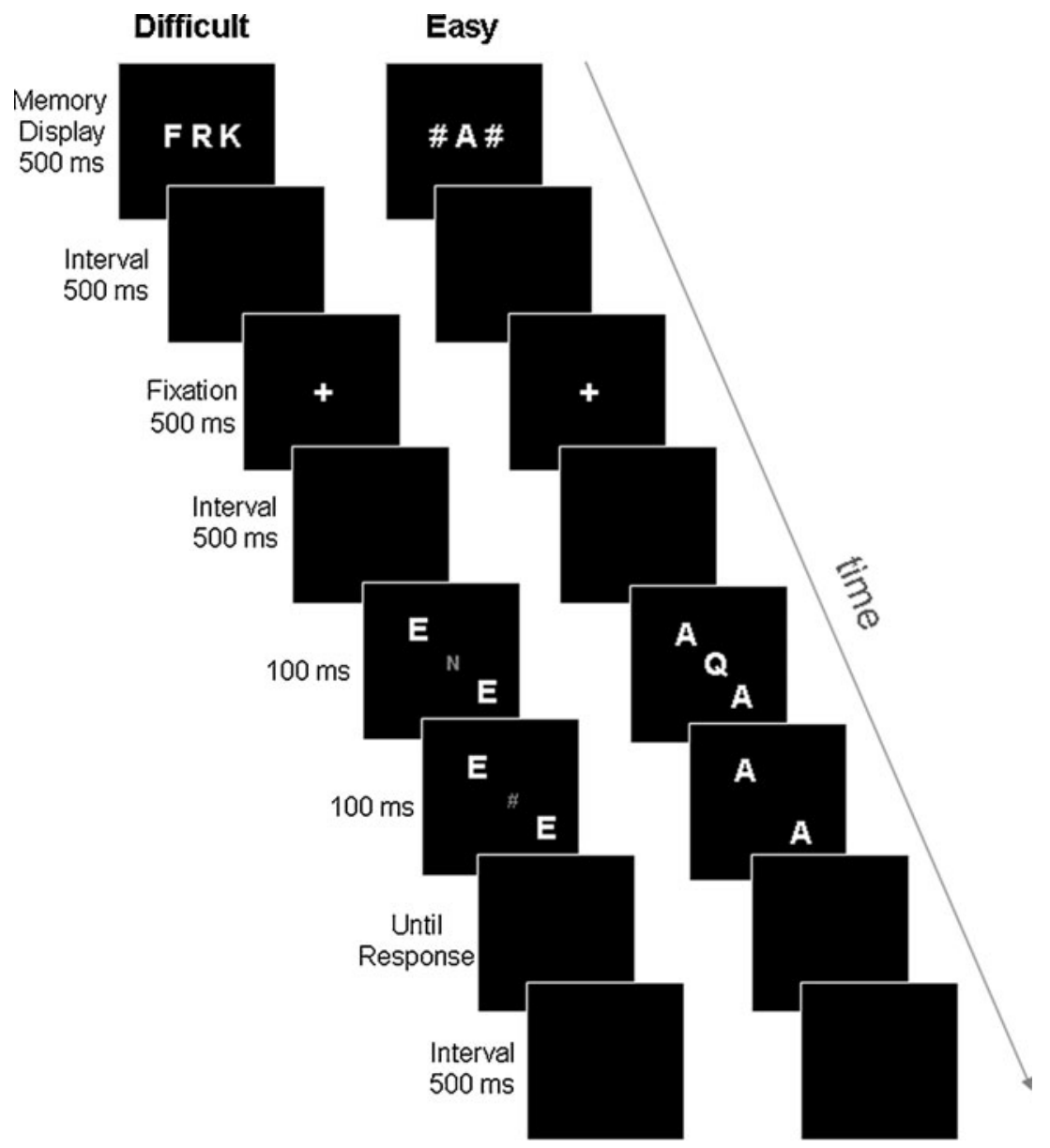


than in easy condition. There was also a borderline effect for congruency $[F(1,9)=4.37, p=.066]$, indicating that congruent trials were less erroneous than incongruent ones. The Condition $\times$ Congruency interaction did not reach statistical significance $[F(1,9)=3.08, p=.1]$.

Incorrect responses and responses deviating by more than two standard deviations from the mean were removed from the RT analyses. The same analysis performed on mean RTs revealed a significant effect of condition $[F(1,9)=10.12, p=$ .011], in which responses in the easy condition were faster than those in the difficult condition, and a significant main effect of congruency $[F(1,9)=7.74, p=.021)$, in which congruent trials were faster than incongruent ones. The Condition $\times$ Congruency interaction, however, did not reach significance $[F(1,9)=0.46, p=.51]$.

The combined load of sensory and memory demands seems to have created a substantial burden in the flanker task. This was evident both in the accuracy measure and in the RT measure. Another indication of the difficulty of the present manipulation can also be seen in the fact that a third of the participants were initially excluded from the analysis due to low accuracy rates in the difficult condition (accuracy rates of the excluded participants in the difficult condition ranged between $51 \%$ and $72 \%$, whereas the accuracy rates in the easy condition ranged between $71 \%$ and $99 \%$ ). In fact, because the difficulty of the task meant that more than a third of our participants were excluded due to high error rates, we conducted the same analysis using the entire participant set, in order to make sure that the reduced set of participants did not skew the response pattern. The results of the full-set analysis mirrored those of the reduced set, with a significant distractor location effect in the temporal judgment task $[F(1,15)=9.45, p<.009]$ and significant condition and congruency effects in both the accuracy measure $[F(1,15)=10.46, p<.007$, for condition; $F(1,15)=$ $4.6, p<.05$, for congruency $]$ and the RT measure $[F(1,15)=$ $7.15, p<.018$, for condition; $F(1,15)=5.22, p<.038$, for congruency] of the flanker task. No other effect reached significance.

The results did not replicate the Load $\times$ Congruency interaction that was found in Experiment 2. This might be due to the present combination of both sensory and memory constraints. Working memory is suggested to control the priority of relevant processing. When working memory is taxed and prioritization is less efficient, attention might be more likely to be drawn toward distractors. If this infiltration of the distractors occurs after the processing of the target, such infiltration is less likely to happen when sensory limitations are involved, because the distractors disappear before they can cause additional interference. Conversely, in Experiment 2, in which the stimulus display remained until response, there was ample distractor data left for increasing the congruency effect, resulting in a significant Load $\times$ Congruency interaction.
Temporal judgment task An overall repeated measures Condition (difficult vs. easy) $\times$ Distractor Location (top left vs. top right) ANOVA was performed on the proportions of "left" responses (see Fig. 6). The analysis revealed that the main effect of distractor location was significant $[F(1,9)=$ $9.2, p=.014]$, showing more "left" responses when the distractor was expected in the top left versus the top right location. Neither the main effect of condition $[F(1,9)=$ $0.51, p=.49]$ nor the interaction between condition and distractor location $[F(1,9)=0.25, p=.63]$ reached statistical significance.

In line with the previous experiments, a significant temporal judgment effect, indicating that attention was directed toward where the distractor was expected to appear, was found in both the easy and difficult conditions. The size of this effect did not change, even with the additional task difficulty. It seems that even when there is an expectation of exceptionally high attentional demands in the upcoming display, a set amount of attention is still always allocated toward the expected distractor locations.

\section{General discussion}

The present results clearly show that severely constraining the perceptual or memory systems did not affect the temporal judgment task; the same amount of attention was allocated to the expected distractor locations under both easy

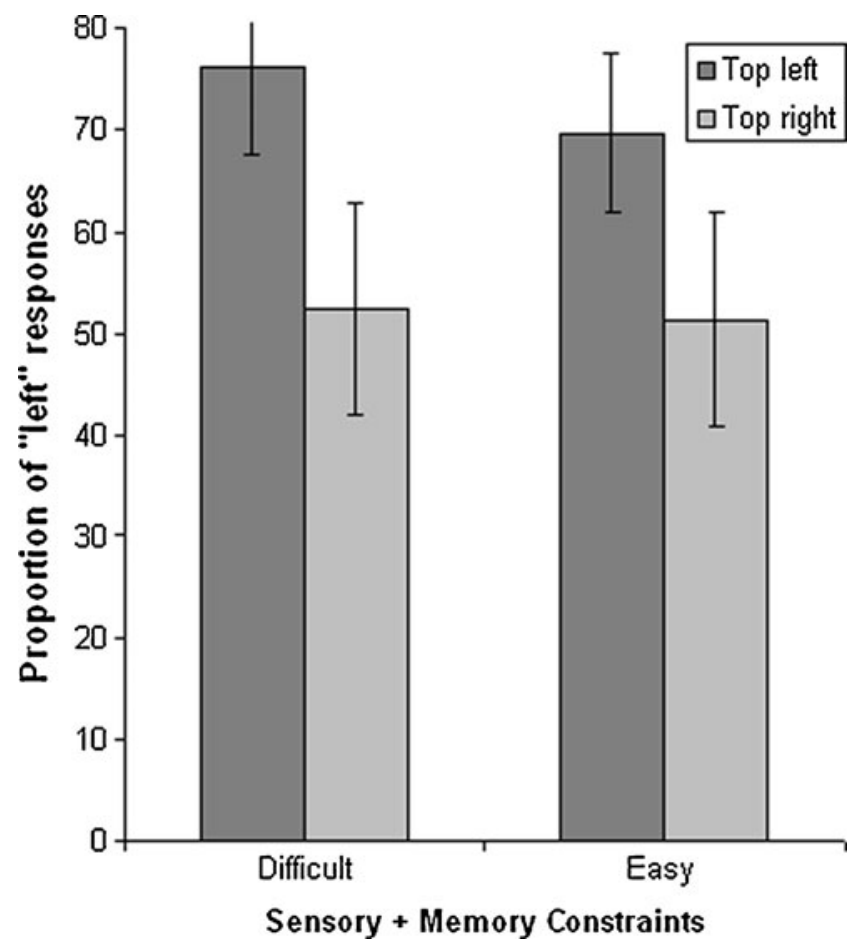

Fig. 6 Mean proportions of "left" responses (perceiving the left dot before the right dot) in the difficult and easy conditions of Experiment 3 
and difficult task conditions. Even when a combination of integrated sensory and memory constraints was utilized, severely taxing the information-processing system, the temporal judgment task was still unaffected, and the amount of attention allocated to the distractor locations remained the same. These results suggest that a fixed amount of attention is always allocated to distractor locations, regardless of expected difficulties and participants' intentions.

\section{Resilience of the AWB effect}

In the present study, we investigated whether attention to distractors can be subject to prioritization by exploring the boundaries of the AWB effect. Delineating the boundaries of this effect could help establish its nature and the attentional processes that mediate the effect. We presented constraints that burdened the flanker task at different levels of processing. The additional constraints required that ample amounts of the available resources be allocated to the processing of the target. The results showed that the decrease in available resources did not come at the expense of the attention allocated to the distractors, suggesting that the AWB effect is mandatory and is unaffected by shifting task prioritization. The fact that the attentional allocation maintained the same magnitude, even when available resources were depleted, suggests that this mandatory attentional allocation is always of a fixed minimal amount.

One may possibly argue that the AWB effect persisted under various types of constraints because the increasing constraints were added only to the flanker task and were not actually present in the temporal judgment display. Thus, the constraints did not affect the AWB effect because they were not present at the time that it was measured. However, this explanation is unlikely, because the AWB effect represents attentional allocation as part of the preparation for the initiation of the trial, and therefore should be affected by the expectations of the upcoming display's difficulty, even if those constraints are not currently encumbering. Indirect support for this claim can be found in the results reported by Theeuwes, Kramer, and Belopolsky (2004), who manipulated the ability to anticipate the level of perceptual load. They showed that when high-load and low-load displays appeared in blocked conditions, congruency effects were found in the low-load but not in the high-load conditions, because participants could anticipate the level of the upcoming load and focus their attention accordingly. However, in mixed conditions, congruency effects were found in both the high- and the low-perceptual-load trials, because expectancy about load level was not possible and attention always had to be spread wide. More specific support for the claim that the reliability of the AWB effect should not be discounted just because the constraints were not present at the time the the temporal bias was measured comes from Experiments 2 and 3 of the present study, in which the additional constraints did pertain to the temporal judgment task as well. In these experiments, the memory display always appeared before the critical temporal judgment or flanker displays, and thus the effects of working memory constraints were already operating at the time that initial attention was allocated, prior to the stimulus display. The results of these experiments revealed that the AWB effect persisted even under the heavy memory constraints, yielding results similar to those of Experiment 1.

The manipulation of a variety of constraints did not alter the AWB effect, leading us to conclude that the effect is mandatory. Evidently, there is always the possibility that the AWB effect may not be completely resilient, but that the methods we used were simply not strong enough to reach its boundaries. We attempted to address this possibility in Experiment 3 by presenting both sensory and memory burdens together. The results of this exceptionally difficult experiment still failed to show a change in the AWB effect. Nevertheless, these are null results, leaving open the possibility that stronger methods could affect the AWB effect. If the AWB effect could indeed be altered by more extreme measures, this effect would be likely to operate in an all-or-none rather than a gradient fashion, since the latter option ought to have been revealed in the various manipulations used in the present study.

\section{The AWB effect versus top-down inhibition}

The present conclusion that advance knowledge of distractor locations results in allocating attention to the distractors appears to conflict with findings that have shown that a probe dot is less likely to be detected in a location that has just been occupied by a distractor than in a previously empty one (e.g., Cepeda, Cave, Bichot, \& Kim, 1998; Chen \& Treisman, 2008). However, these seemingly opposing claims need not exclude each other, but rather reflect diverse effects occurring at different points in the informationprocessing flow. The finding that more attention is allocated to empty spaces than to distractor locations may reflect laterstage response inhibition rather than perceptual inhibition of the distractor, since the probe used to measure the distribution of attention appeared after stimulus presentation. Indeed, the results of a study by Humphreys, Stalmann, and Olivers (2004) suggested that the locations of distractor items are initially attended at an early processing stage, before they can be actively inhibited. In their study, a probe dot was presented at the distractor location at shorter and longer intervals following the appearance of the distractor but preceding the appearance of the target and additional, new distractors. The results showed that $200 \mathrm{~ms}$ after the initial presentation of the distractors, a probe dot presented at a distractor location was more likely to be detected than a 
probe presented at an empty location. At longer intervals $(800 \mathrm{~ms})$, this pattern was reversed, so that probes presented at distractor locations were less likely to be detected than those presented at empty locations. Taken together, these results suggest that selective processing of stimuli at distractor locations changes during the time course of a trial: Prior to stimulus presentation, these locations are enhanced, whereas following the presentation of the target display, they are inhibited. Perhaps this process explains how the conflict between the target and incompatible distractors in flanker displays is eventually resolved. That is, the fact that distractor interference subsides over time might allow the response mapped to the target identity to be ultimately chosen and executed.

In a number of studies, researchers have examined what happens to distractors when participants are informed of their locations prior to the appearance of the display, showing that such preknowledge actually produces perceptual inhibition at the distractor locations (e.g., Chao, 2010; Munneke, Van der Stigchel, \& Theeuwes, 2008; Ruff \& Driver, 2006). These studies have shown that target processing is improved when distractor locations are known in advance, leading to the conclusion that distractor locations are inhibited. However, it is not clear whether these experiments demonstrated inhibition of known distractor locations, or rather a strategy of ignoring a subset of the search display. For example, in Munneke et al.'s study, four possible stimulus locations were used. On half of the trials the distractor location was precued, and participants thus knew that the target was to appear in one of the remaining three locations. It is therefore possible that on these trials, participants allocated attention to three rather than four possible expected locations, thereby producing improved performance. Thus, improved performance on distractor-preknown trials may reflect a denser distribution of attention over expected target locations rather than the active inhibition of an expected distractor location. In fact, studies that did not permit the omitting of a cued distractor location from the search array have not shown any indication of inhibition. In Chao (2010), as well as in Buckolz, Guy, Khan, and Lawrence (2006), participants were cued with one of four display locations as the location of the distractor with $67 \%$ validity. The target could still appear in any of the four display locations with equal probability. The results showed that performance was not affected by distractor precuing, suggesting that the distractor was not inhibited. This experimental design hindered participants' ability to use the information from the cue to shift attention away from the distractor, because all locations remained relevant for the target. Hence, when attention cannot be shifted away from the location of the distractor, there is no indication of distractor inhibition.

\section{References}

Baddeley, A. (1986). Working memory. Oxford, U.K.: Oxford University Press, Clarendon Press.

Buckolz, E., Guy, S., Khan, M., \& Lawrence, G. (2006). Can the location negative priming process operate in proactive manner? Psychological Research, 70, 218-227.

Cave, K. R., \& Wolfe, J. M. (1990). Modeling the role of parallel processing in visual search. Cognitive Psychology, 22, 225-271. doi:10.1016/0010-0285(90)90017-X

Cepeda, N. J., Cave, K. R., Bichot, N. P., \& Kim, M.-S. (1998). Spatial selection via feature-driven inhibition of distractor locations. Perception \& Psychophysics, 60, 727-746. doi:10.3758/ BF03206059

Chao, H.-F. (2010). Top down attentional control for distractor locations: The benefit of precuing distractor locations on target localization and discrimination. Journal of Experimental Psychology: Human Perception and Performance, 36, 303-316.

Chen, Z., \& Treisman, A. (2008). Distractor inhibition is more effective at a central than at a peripheral location. Perception \& Psychophysics, 70, 1081-1091.

Desimone, R., \& Duncan, J. (1995). Neural mechanisms of selective visual attention. Annual Review of Neuroscience, 18, 193-222. doi:10.1146/annurev.ne.18.030195.001205

Downing, P. E. (2000). Interactions between visual working memory and selective attention. Psychological Science, 11, 467-473. doi:10.1111/1467-9280.00290

Eltiti, S., Wallace, D., \& Fox, E. (2005). Selective target processing: Perceptual load or distractor salience? Perception \& Psychophysics, 67, 876-885. doi:10.3758/BF03193540

Eriksen, B. A., \& Eriksen, C. W. (1974). Effects of noise letters upon the identification of a target letter in a nonsearch task. Perception \& Psychophysics, 16, 143-149. doi:10.3758/BF03203267

Hikosaka, O., Miyauchi, S., \& Shimojo, S. (1993). Focal visual attention produces illusionary temporal order and motion sensation. Visual Research, 33, 1219-1240. doi:10.1016/0042-6989(93) 90210-N

Humphreys, G. W., Stalmann, B. J., \& Olivers, C. (2004). An analysis of the time course of attention in preview search. Perception \& Psychophysics, 66, 713-730.

Lavie, N. (1995). Perceptual load as a necessary condition for selective attention. Journal of Experimental Psychology: Human Perception and Performance, 21, 451-468.

Lavie, N., \& de Fockert, J. (2005). The role of working memory in attentional capture. Psychonomic Bulletin \& Review, 13, 669-674.

Lavie, N., Hirst, A., de Fockert, J. W., \& Viding, E. (2004). Load theory of selective attention and cognitive control. Journal of Experimental Psychology: General, 133, 339-354. doi:10.1037/ 0096-3445.133.3.339

Makovski, T., Swallow, K. M., \& Jiang, Y. V. (2011). Attending to unrelated targets boosts color short-term memory for color arrays. Neuropsychologia, 49, 1498-1505.

Miller, J. (1991). The flanker compatibility effect as a function of visual angle, attentional focus, visual transients, and perceptual load: A search for boundary conditions. Perception \& Psychophysics, 49, 270-288. doi:10.3758/BF03214311

Munneke, J., Van der Stigchel, S., \& Theeuwes, J. (2008). Cueing the location of a distractor: An inhibitory mechanism of spatial attention? Acta Psychologica, 129, 101-107.

Norman, D. A., \& Bobrow, D. G. (1975). On data-limited and resource limited processes. Cognitive Psychology, 7, 44-64.

Olivers, C. N. L. (2009). What drives memory-driven attentional capture? The effects of memory type, display type, and search type. Journal of Experimental Psychology: Human Perception and Performance, 35, 1275-1291. 
Ruff, C. C., \& Driver, J. (2006). Attentional preparation for a lateralized visual distractor: Behavioral and fMRI evidence. Journal of Cognitive Neuroscience, 18, 522-538.

Shore, D. I., Spence, C., \& Klein, R. M. (2001). Visual prior entry. Psychological Science, 12, 205-212. doi:10.1111/14679280.00337

Soto, D., Heinke, D., Humphreys, G. W., \& Blanko, M. J. (2005). Early, involuntary top-down guidance of attention from working memory. Journal of Experimental Psychology: Human Perception and Performance, 31, 248-261.

Stelmach, L. B., \& Herdman, C. M. (1991). Direct attention and perception of temporal order. Journal of Experimental Psychology: Human Perception and Performance, 17, 539-550. doi:10.1037/ 0096-1523.17.2.539

Theeuwes, J., Kramer, A. F., \& Belopolsky, A. V. (2004). Attentional set interacts with perceptual load in visual search. Psychonomic Bulletin \& Review, 11, 697-702. doi:10.3758/BF03196622
Treisman, A. M., \& Gelade, G. (1980). A feature-integration theory of attention. Cognitive Psychology, 12, 97-136. doi:10.1016/00100285(80)90005-5

Tsal, Y., \& Benoni, H. (2010). Diluting the burden of load: Perceptual load effects are simply dilution effects. Journal of Experimental Psychology: Human Perception and Performance, 36, 16451656.

Tsal, Y., \& Makovski, T. (2006). The attentional white bear phenomenon: The mandatory allocation of attention to expected distractor location. Journal of Experimental Psychology: Human Perception and Performance, 32, 351-363. doi:10.1037/0096-1523.32.2.351

Wilson, D. E., Muroi, M., \& MacLeod, C. M. (2011). Dilution, not load, affects distractor processing. Journal of Experimental Psychology: Human Perception and Performance, 37, 319-335.

Wolfe, J. M. (1994). Guided Search 2.0: A revised model of visual search. Psychonomic Bulletin \& Review, 1, 202-238. doi:10.3758/ BF03200774 